\title{
Rejuvenating Tithonus
}

\author{
Kyle M. Loh \& Bing Lim
}

$\mathrm{T}$ ennyson tells us of Tithonus, who was endowed with immortality and was never permitted to perish, despite his wishes to that end. A biological parallel might be pluripotent stem cells, which can be indefinitely propagated in vitro in an uncommitted, self-renewing state, and can form all lineages in the mammalian body [1]. However, pluripotency is an inherently transient condition in vivo. Thus, pluripotent cells in vitro, such as embryonic stem cells (ESCs), continually show proclivities towards differentiation, similarly to Tithonus seeking the termination of his immortality [1]. To counteract this, several molecular actors, including the transcription factor $\operatorname{Prdm14}$, within self-renewing pluripotent cells restrain forward developmental progression $[2,3]$. Four papers, including one in this issue of EMBO reports, depict mechanisms by which $\operatorname{Prdm} 14$ serves as the elixir of youth for pluripotent stem cells [4-7], acting at the levels of developmental signalling and chromatin.

\section{Four papers, including one in this issue of EMBO reports, depict mechanisms by which $\operatorname{Prdm} 14$ serves as the elixir of youth for pluripotent stem cells...}

At the level of signalling, Prdm14 desensitizes pluripotent cells to differentiationinducing extrinsic signals. In vivo, the pluripotent cells of the blastocyst-stage epiblast-from which ESCs are derivedexpress Fgf4, which sustains adjacent extra-embryonic endoderm cells. However, endogenous fibroblast growth factor (FGF) signalling within ESC cultures in vitro promotes disassembly of the undifferentiated state and directs commitment to extraembryonic or primitive streak lineages [8]. Prdm14 safeguards ESCs from autocrine FGF signalling by binding to and downregulating expression of Fgfr genes [5,7], apparently through the repression of distal enhancer elements that control Fgfr expression. Genetic removal of Prdm14 renders ESCs more susceptible to the inductive effects of FGF signalling [5,7]. Yet, $\operatorname{Prdm} 14$

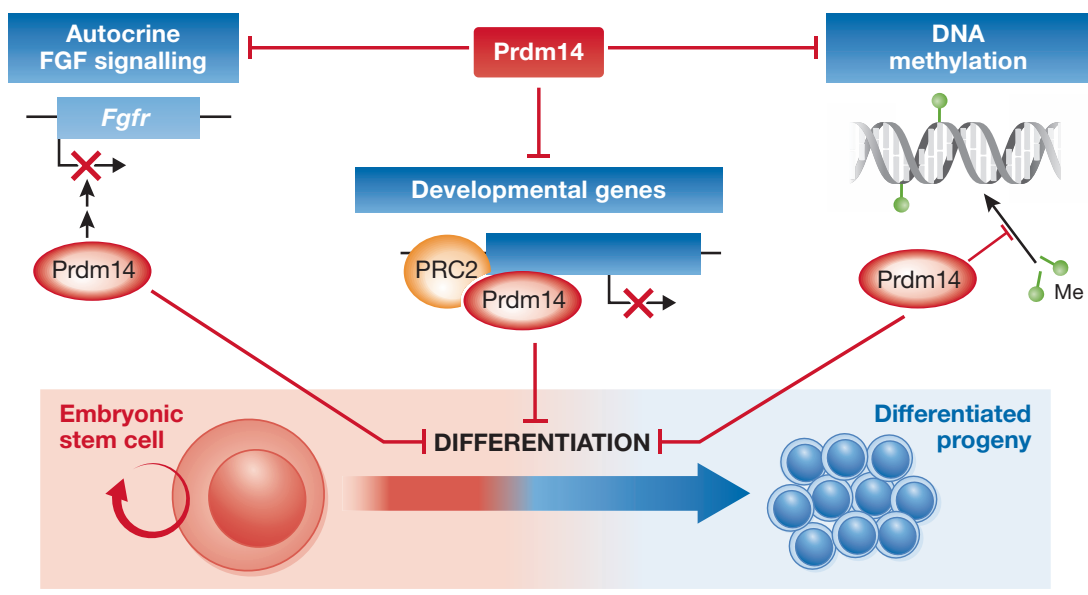

Fig 1 | Prdm14 represses autocrine FGF signalling, occupies and suppresses multiple differentiationassociated genes and globally suppresses DNA methylation. This collectively restricts differentiation and entraps pluripotent cells in an uncommitted state. FGF, fibroblast growth factor.

acts more broadly than just counteracting the effects of FGF signalling, because epistatic experiments show that even if FGF/ MAPK (mitogen-activated protein kinase) signalling is pharmacologically inhibited, Prdm 14 is still important for undifferentiated ESC self-renewal $[5,7]$.

To identify the multiplicity of targets through which Prdm14 might act, two studies used chromatin immunoprecipitation followed by sequencing to systematically chart genes across the genome bound by Prdm14 [2,3]. By combining this cis-regulatory data with gene expression changes after perturbing Prdm14 expression, two more studies found that Prdm14 directly occupies a variety of regulatory genes that control germ layer formation and anterior-posterior patterning-for example, Nodal, Snai1 and Hhex-and downregulates their expression [5,7]. How does Prdm14 prevent activation of these developmental programmes?

Prdm14 physically interacts with polycomb repressive complex 2 (PRC2; $[4,7]$ ), a chromatin-modifying complex that suppresses gene transcription and deposits the H3K27me3 histone modification. This suggests that Prdm14 targets PRC2 to developmental genes to inhibit their expression. Indeed, transcriptional and chromatin state analyses have revealed that on genetic ablation of Prdm14, PRC2 and H3K27me3 fail to accumulate at archetypic Prdm14 target genes $[4,5]$. Thus, in the absence of $\operatorname{Prdm} 14$, differentiation-associated transcriptional programmes are activated to some extent in undifferentiated ESC.

Reports also suggest that Prdm14 forestalls differentiation by globally attenuating DNA methylation [5-7]. DNA methylation, which is often associated with transcriptional repression, is required for differentiation but is dispensable for the self-renewal of undifferentiated cells [9]. Accordingly, various pluripotent cell types are generally characterized by genome-wide DNA hypomethylation [6]. Prdm14 impedes DNA methylation in ESCs, apparently at a global level, as $\operatorname{Prdm} 14$ deletion leads to a widespread increase of DNA methylation. Prdm 14 broadly represses DNA methylation by binding to and downregulating the expression of DNA methyltransferase genes, such as Dnmt3a and Dnmt3b [5-7].

Prdm14 thus pre-empts the precocious differentiation of pluripotent cells through a variety of molecular means. However, simply assigning Prdm14 the role of a general antagonist of differentiation is an

\section{...Prdm14 desensitizes pluripotent cells to differentiation-inducing extrinsic signals}


oversimplification. The particular lineage choices that Prdm14 represses remain unclear, as pluripotency factors can often specifically inhibit particular prospective developmental fates whilst promoting others [1]. More broadly, the phenotypic importance of Prdm 14 remains controversial. In typical serum-containing culture conditions for ESC propagation, ESCs deteriorate if $\operatorname{Prdm} 14$ is genetically ablated. Yet, within ' $2 \mathrm{i}$ ' conditions, in which differentiation-inducing signalling is minimized, Prdm $14^{-/}$ESCs still thrive despite modest upregulation of differentiation genes and a plethora of other defects $[5,7]$. Thus, if differentiation signals are withdrawn, ESCs become less reliant on Prdm 14. Similarly, Prdm $14^{-/-}$mice do not show peri-implantation lethality - the typical consequence of disrupting pluripotent epiblast formation in vivo-rather, they

\section{Prdm14 impedes DNA methylation in ESCs [...] at a global level [...] by binding to and downregulating the expression of DNA methyltransferase genes}

have later deficiencies in germline development [10]. Perhaps Prdm14 suppresses a battery of developmental genes by recruiting PRC2, but when extrinsic activating signals are minimized, these differentiationassociated genes cannot be brought into play, and Prdm14 becomes less important. Equally, other pluripotency factors-for example, Esrrb_-are dispensable for self-renewal if serum is withdrawn [11], suggesting that some pluripotency factors largely serve to dampen differentiation genes otherwise induced by serum, and thus serve in a more ancillary capacity. In any case, development in vivo is accompanied by Prdm14 downregulation [10] and persistent Prdm14 expression perturbs proper differentiation [5]. How developmental morphogens suppress Prdm14 to support differentiation remains a pertinent question, the answer to which is how, at last, Tithonus might be laid to rest.

\section{CONFLICT OF INTEREST}

The authors declare that they have no conflict of interest.

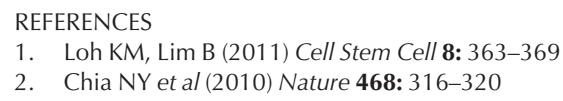

3. Ma Z et al (2011) Nat Struct Mol Biol 18: 120-127

4. Chan YS et al (2012) Stem Cells 31: 682-692

5. Grabole N et al (2013) EMBO Rep [Epub ahead of print] doi:10.1038/embor.2013.67

6. Leitch HG et al (2013) Nat Struct Mol Biol 20: 311-316

7. Yamaji M et al (2013) Cell Stem Cell 12: 368-382

8. Kunath T et al (2007) Development 134: 2895-2902

9. Tsumara A et al (2006) Genes Cell 11: 805-814

10. Yamaji M et al (2008) Nat Genet 40: 1016-1022

11. Martello G et al (2012) Cell Stem Cell 11: 491-504

Kyle M. Loh is at the Department of Developmental Biology, Stanford University School of Medicine, and the Stanford Institute for Stem Cell Biology \& Regenerative Medicine, Stanford, California, USA.

Bing Lim is at the Genome Institute of Singapore, Stem Cell \& Developmental Biology Group, Singapore, and the Department of Haematology/Oncology, Beth Israel Deaconess Medical Centre and Harvard Medical School, Boston, Massachusetts, USA.

E-mail: limb1@gis.a-star.edu.sg

EMBO reports (2013) 14, 583-584; published online 14 June 2013; doi:10.1038/embor.2013.81 